\title{
Superhydrophobic Paper in the Development of Disposable Labware and Lab-on-Paper Devices
}

\author{
Maria Peixoto Sousa ${ }^{\dagger+\ddagger}$ and João Filipe Mano ${ }^{\dagger, \ddagger} *$ \\ ${ }^{\dagger}$ 3B Research Group - Biomaterials, Biodegradables and Biomimetics, School of Engineering, University of Minho, Avepark, Zona \\ Industrial da Gandra, S. Claudio do Barco, 4806-909 Caldas das Taipas, Guimarães, Portugal \\ "ICVS/3B’s - PT Government Associate Laboratory, Braga/Guimarães, Portugal
}

\begin{abstract}
Traditionally in superhydrophobic surfaces history, the focus has frequently settled on the use of complex processing methodologies using nonbiodegradable and costly materials. In light of recent events on lab-on-paper emergence, there are now some efforts for the production of superhydrophobic paper but still with little development and confined to the fabrication of flat devices. This work gives a new look at the range of possible applications of bioinspired superhydrophobic paper-based substrates, obtained using a straightforward surface modification with poly(hydroxybutyrate). As an end-of-proof of the possibility to create lab-on-chip portable devices, the patterning of super-

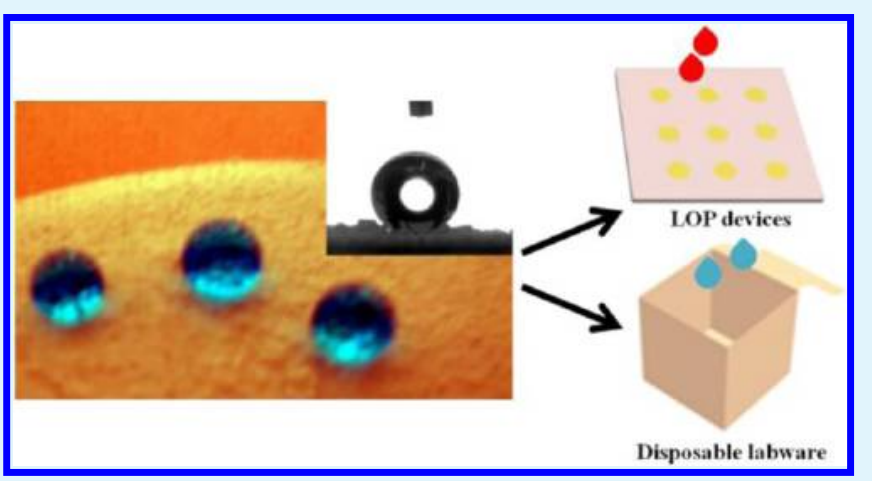
hydrophobic paper with different wettable shapes is shown with low-cost approaches. Furthermore, we suggest the use of superhydrophobic paper as an extremely low-cost material to design essential nonplanar lab apparatus, including reservoirs for liquid storage and manipulation, funnels, tips for pipettes, or accordion-shaped substrates for liquid transport or mixing. Such devices take the advantage of the self-cleaning and extremely water resistance properties of the surfaces as well as the actions that may be done with paper such as cut, glue, write, fold, warp, or burn. The obtained substrates showed lower propensity to adsorb proteins than the original paper, kept superhydrophobic character upon ethylene oxide sterilization and are disposable, suggesting that the developing devices could be especially adequate for use in contact with biological and hazardous materials.
\end{abstract}

KEYWORDS: superhydrophobic surface, lotus-effect, lab-on-paper, paper labware, protein adsorption, origami

\section{INTRODUCTION}

Since paper's origin, new ways of using it have been devised day-by-day. Today, the range of possibilities seems practically unlimitable; indeed paper can be written or printed on, but cut, folded, coated, embossed, colored, packed, and so on. Thanks to its low-cost, biodegradability, wide availability, and ability to modify and functionalize cellulose fibers, paper has been recognized as an appealing substrate to be applied for more technical and specialized purposes. ${ }^{1-5}$ Recent literature suggests their interest in microfluidic devices for biochemical analysis or less expensive health and point-of-care (POC) diagnosis, ${ }^{6-8}$ MEMS systems, ${ }^{9}$ and two-dimensional (2D) systems to transport, mix, and store liquid samples. ${ }^{10,11}$ In particular, following the innovative studies of Whitesides' group, ${ }^{12-14}$ many attempts have been made to obtain patterned paper-based devices, most based on the same working principle: the hydrophobic-hydrophilic contrast. ${ }^{1}$

In the last years, many processing methodologies have been proposed to produce patterned paper microfluidic devices, mostly exploring photolithography, ${ }^{12,15}$ plasma treatment, ${ }^{16,17}$ inkjet printin, $\mathrm{g}^{18}$ and wax printing ${ }^{19,20}$ approaches. However, little research has focused yet on the use of superhydrophobic (SH) paper. ${ }^{21-24}$ We recently suggested a simple methodology to prepare bioinspired rough $\mathrm{SH}$ paper surfaces through a simple surface modification using poly(hydroxybutyrate), PHB: the resulting substrates exhibited apparent water contact angle (WCA) of $153.0 \pm 0.7^{\circ} .{ }^{25}$ The $\mathrm{SH}$ paper may bring new strengths on the development of lab-on-paper (LOP) devices: the increase in the wettability contrasts between wettable and repellent regions, the prevention of water and moisture absorption, and the avoidance of pathogenic contamination. $^{22-24,26}$

This work proposes different methods to pattern flat $\mathrm{SH}$ paper surfaces creating wettable domains that contrast with the superhydrophobic behavior of the remaining area.

Currently, the design of LOP devices are mainly achieved by patterning techniques. Because Whitesides and their co-workers reported the use of photoresist agents activated by a UV light to pattern the surface of chromatographic paper with more hydrophobic domains, ${ }^{12}$ faster and straightforward techniques have been conceived. For instance, by using a standard inkjet printer Whitesides ${ }^{13}$ achieved a more efficient route to pattern

Received: January 25, 2013

Accepted: April 12, 2013

Published: April 12, 2013 


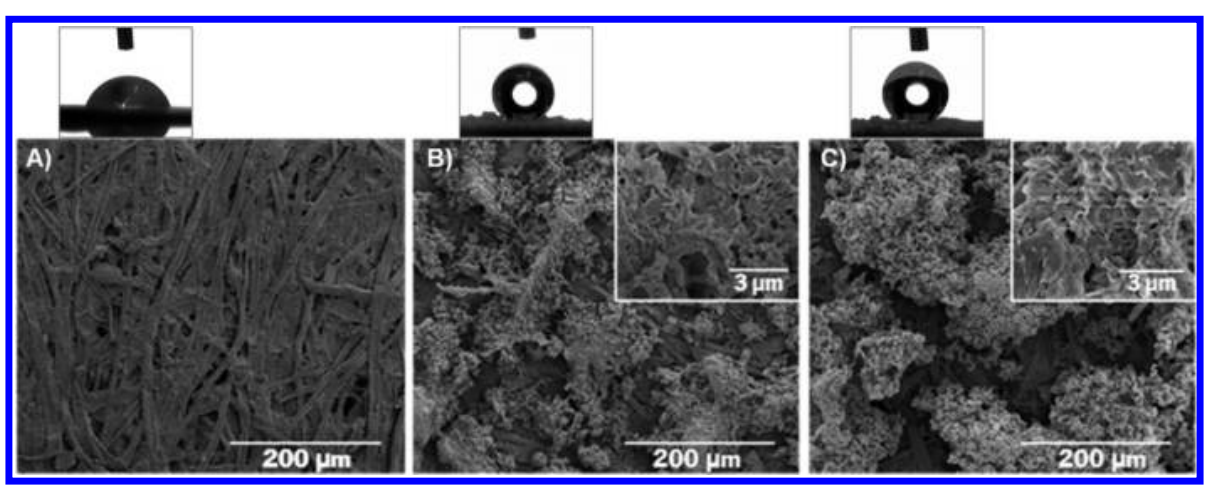

Figure 1. Representative SEM images of (A) commercial cartridge paper used to prepare the substrates, (B) the surface of $\mathrm{SH}$ paper, and (C) SH paper after ethylene oxide sterilization. The inset images are magnified SEM pictures. The top images show representative profiles of $3 \mu \mathrm{L}$ water droplets over the surfaces.

the paper substrate with regions with distinct wettabilities; Lu et al. $^{20}$ developed a microfluidic paper-based analytical device by drawing different patterned geometries with a wax crayon or with a printer, followed by a heating step to melt the wax. We hypothesize that better results could be produced using $\mathrm{SH}$ substrates, creating a well-marked contrast with higher definition and increase the stability of the liquid dispensed in the wettable domains. It is widely known that processes such plasma treatment and UV irradiation are able to modify chemically rough $\mathrm{SH}$ surfaces, just by adjusting the exposure time. $^{27,28}$

Three-dimensional (3D) paper-based systems are suggested in the literature: Martinez et al. ${ }^{14}$ reported the fabrication of a multilayered structure composed by single layers of patterned photoresist paper stacked one above the other for biochemical detection; Liu and Crooks ${ }^{29}$ developed an origami-inspired patterned paper which can be unfolded for parallel chemical analysis. Ge et al. ${ }^{30}$ described a wax-patterned $3 \mathrm{D}$ paper-based electrochemiluminescence immunoassay to detect tumor markers. In this work, we also intend to extend the use of paper to produce $3 \mathrm{D}$ devices by exploring the self-cleaning and extreme water repellent properties of the developed $\mathrm{SH}$ paper. In particular, we consider that $\mathrm{SH}$ paper would be profitable to reproduce conventional laboratory devices. Plastic (mainly polystyrene, polypropylene, and polycarbonate) and glass materials are widely used by labware industry to produce beakers, eppendorf, disposable tips, pipettes, and so on. ${ }^{31}$ Effectively, there is a lack of low-cost and disposable alternatives to these materials, mainly for resource-limited laboratories. We demonstrate in this work that $\mathrm{SH}$ paper, being flexible, inexpensive, safe, and incinerable, could be an alternative low carbon footprint material for such kind of applications.

\section{MATERIALS AND METHODS}

2.1. Materials. The cartridge paper sheets, used for the development of paper-based substrates, were purchased from Pontus. This type of paper distinguishes of common paper by its higher density $\left(120 \mathrm{~g} / \mathrm{cm}^{3}\right)$, allowing greater resistance to handling. Commercial grade poly(hydroxybutyrate), $\mathrm{PHB}$, with a melting point of $173{ }^{\circ} \mathrm{C}$, was kindly provided by Biomer in the form of a very thin powder. Chloroform and absolute ethanol, with $99 \%$ and 99.5\% of purity, were supplied by Carlo Erba Reagent and Pancreac, respectively.

2.2. Methods. 2.2.1. Preparation of Superhydrophobic Paper. $\mathrm{SH}$ paper samples were prepared based on a process proposed before. ${ }^{25}$ Paper samples with different sizes and shapes were cut from A4 sheets of cartridge paper and then immersed in chloroform for at least $6 \mathrm{~h}$, to extract any possible additives soluble in the solvent. The pieces of paper were then immersed for a period of $6 \mathrm{~h}$ into a $7.5 \%$ $(w / v)$ solution of PHB in chloroform. Subsequently, a new immersion was carried out into a coagulation bath, composed by a mixture of $85 /$ $15(\mathrm{v} / \mathrm{v})$ of ethanol and water, for $12 \mathrm{~h}$. The resulting samples were then placed in a glass surface and dried at room temperature and atmospheric pressure.

2.2.2. Characterization Techniques. Scanning Electron Microscopy (SEM). The morphology and the topography of samples surface were analyzed by SEM, using a Leica Cambridge S-360 SEM (Leica Cambridge, U.K.). The surfaces were sputter-coated before with a thin gold layer.

Protein Adsorption Quantification - BCA Assay. Through the use of a bicinchoninic acid (BCA) protein assay kit, supplied by Pierce Chemical Co (USA), we could quantify the protein absorption onto the surfaces of both original and $\mathrm{SH}$ paper by a simple colorimetric detection method. The operating principle is based on use of BCA to detect $\mathrm{Cu}^{1+}$. In the presence of a protein and an alkaline environment, $\mathrm{Cu}^{2+}$ is reduced to $\mathrm{Cu}^{1+}$, providing a purplelike reaction. $\mathrm{Cu}^{1+}$ displays a strong absorbance at $562 \mathrm{~nm}$, showing a linear behavior with the rise of protein concentration.

Circular samples $(10 \mathrm{~mm})$ of common and $\mathrm{SH}$ cartridge paper were carefully fixed on the bottom of an ultra low attachment 12-well plates and immersed in $3 \mathrm{~mL}$ of five different concentrations of bovine serum albumin (BSA, Sigma-Aldrich) in phosphate buffered saline (PBS, Gibco): 7, 16, 32, 78, 150, and $260 \mu \mathrm{g} \mathrm{mL}^{-1}$. The analysis was made after $1 \mathrm{~h}$ of BSA adsorption. The remaining protein in solution was evaluated, according with the provider's instructions. The protein concentration was determinated by the measure of the absorbance using a calibration curve for BSA, established using a series of protein solutions with known concentrations. The absorbance was read on a microplate spectrophotometer (Tecan), at $562 \mathrm{~nm}$. The amount of protein absorbed was calculated for each sample subtracting the obtained value of microplate reader from the initial value of protein put in contact with the substrate.

Sterilization Procedure. To entirely fulfill the requisites of general laboratorial material, the $\mathrm{SH}$ paper surfaces must be resistant to at least one practical sterilization technique. Squares of common, $\mathrm{SH}$ and patterned paper with $1 \mathrm{~cm}^{2}$ of surface area were sterilized by ethylene oxide at Pronefro (Portugal). Standard conditions involve a working temperature of $45^{\circ} \mathrm{C}$, a cycle time of $14 \mathrm{~h}$, a chamber pressure of 50 $\mathrm{kPa}$ and a humidity level of $50 \%$.

Apparent Water Contact Angle (WCA). The wettability of the prepared surfaces was evaluated by WCA measurements, using an OCA15+ goinometer from DataPhysics (Germany), at room temperature, with a conventional sessile drop method. A $3 \mu \mathrm{L}$ droplet of HPLC grade water was deposited on the surface. The shape of each droplet was recorded and analyzed using the SCA 20 software, $15 \mathrm{~s}$ after the drop deposition. At least five measurements were taken for each sample.

2.2.3. Patterning of Two-Dimensional (2D) Superhydrophobic Surfaces. To modify the wettability of the samples, we used a ProCleaner 220 (BioForce Nanoscience, USA) to treat the surface 
with a UV/ozone (UVO) irradiation. The exposure time ranged between 1 and $30 \mathrm{~min}$. After the treatment, all the substrates were stored in a dry environment until characterization. Distinct geometries were patterned on the samples surface, emphasizing the multifaceted ability to create low-cost designs. For the UVO treatment we used acetate polymeric sheets that were cut with desirable hollowed geometrical features. By putting such masks over the SH paper, the cut-out part of the acetate sheet exposes confined regions of the sample to the UVO light, increasing the wettability in just these regions.

Also writing, printing, and stick-on flat objects are here considered as innovative tools to modify and pattern the SH paper surface. We investigated simple water-based markers (Uso), stick-on circles (Uso), and printing models to pattern the substrates with hydrophilic domains. The printing models were developed in Word 2007 software and printed in a standard and commercially available inkjet printer (Epson Stylus SX105).

We used standard areas $\left(9\right.$ and $16 \mathrm{~cm}^{2}$ ) to prepare $\mathrm{SH}$ paper structures. The photos were taken by a Panasonic photographic digital camera (Lumix FS14).

2.2.4. 3D Superhydrophobic Paper Architectures. Different structures were engineered using the developed $\mathrm{SH}$ paper, in order to reproduce some conventional labware, that can be obtained manually by basic origami or cut/glue methods.

2.2.5. Statistical Analysis. All data are described as a mean \pm standard deviation. Statistical significance was analyzed using the oneway ANOVA test, with $p<0.05$ considered differences as statistically significant.

\section{RESULTS AND DISCUSSION}

3.1. Characterization of the Superhydrophobic Paper Surface. SEM Analysis. Changes in the topographical structure

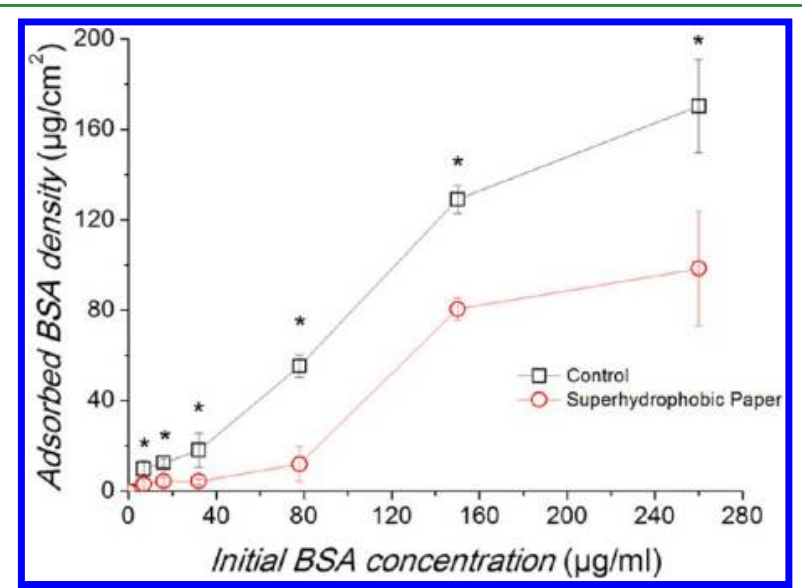

Figure 2. BSA adsorption after $1 \mathrm{~h}$ of incubation in solutions with different initial protein concentrations, comparing rough $\mathrm{SH}$ paper (circles) and commercial cartridge paper (squares). ${ }^{*}$ The differences for the same initial BSA concentration are statistically significant $(p \leq$ $0.005)$.

of paper surface using the proposed methodology were confirmed by SEM. Paper is composed by smooth cellulose fibers, see Figure 1A. PHB, dissolved in chloroform, was put in contact with paper surface, penetrating into the randomly organized cellulose fibers. When immersed into the coagulation bath, the nonsolvent started to diffuse into the polymer solution; as ethanol and chloroform are miscible, the exchanges between solvent and nonsolvent beyond the paper surface resulted in a thermodynamic instability of the system. ${ }^{32-35}$ To attain the minimum Gibb's free energy, the system replies with phase separation of $\mathrm{PHB}$, separating in both poor and rich polymer phases. ${ }^{32-34,36}$ Particular rough structures were

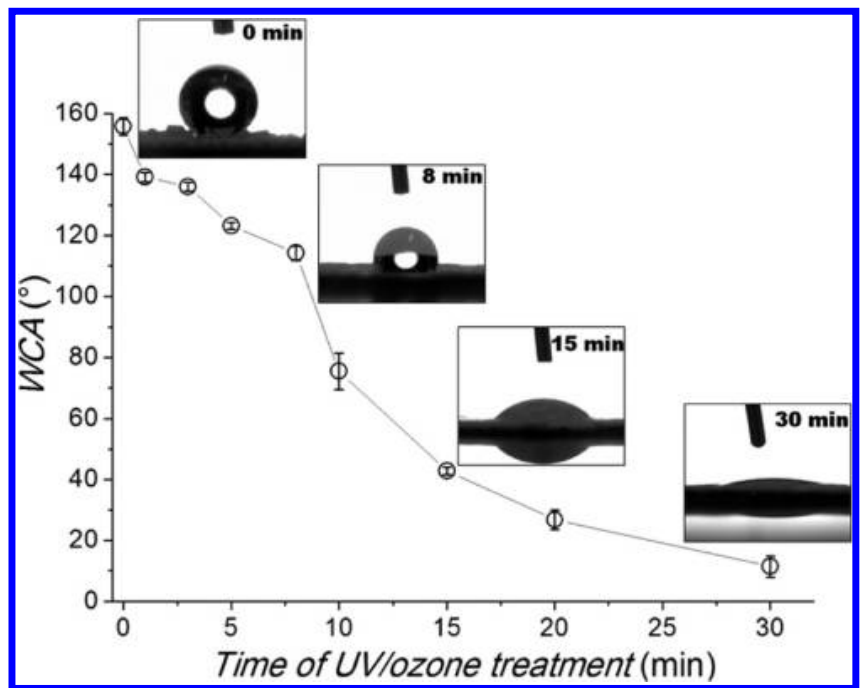

Figure 3. WCA of a $3 \mu \mathrm{L}$ water droplet dispensed on initially $\mathrm{SH}$ paper treated with UVO irradiation for different times (in minutes). Micrographs showing the profiles of $3 \mu \mathrm{L}$ water droplets over the surfaces are shown for specific time-points.

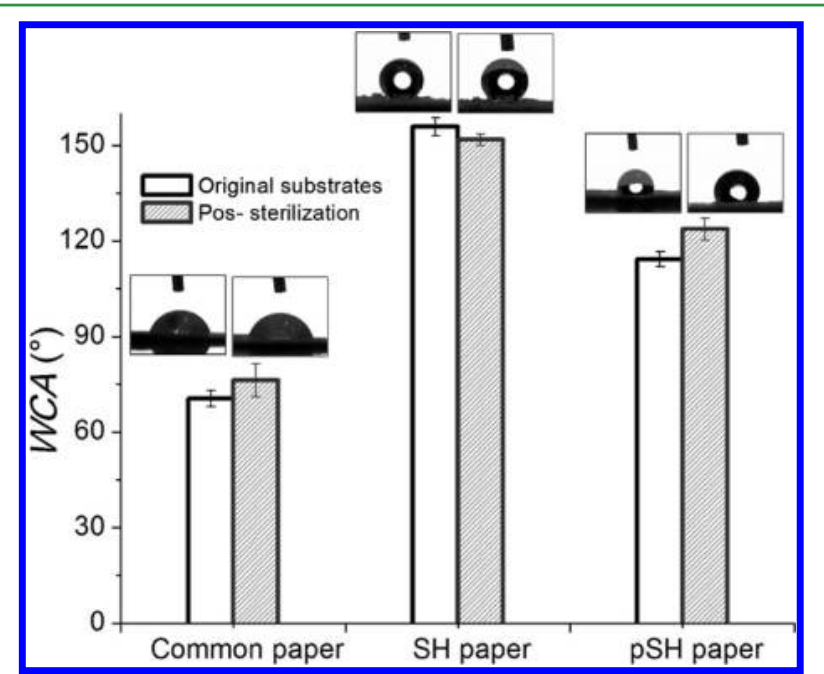

Figure 4. WCA of $3 \mu \mathrm{L}$ water droplets dispensed on different substrates: comparison between non sterilized and ethylene oxide sterilized commercial cartridge paper (Common paper), SH paper and $\mathrm{SH}$ paper treated with $8 \mathrm{~min}$ of UVO irradiation (pSH paper). The graphic contains the picture of the profiles of water droplets over the surfaces, for each cases.

generated: the precipitation of $\mathrm{PHB}$-poor phase resulted in the disperse polymer nuclei and the precipitated PHB-rich phase formed the polymer matrix, aggregating around these nuclei. ${ }^{37,38}$ The nanoarchitectures may be a consequence of the continuous deposition of $\mathrm{PHB}$, precipitated in the rich phase see Figure 1B.

Common cartridge paper exhibited a WCA of $70.5 \pm 2.51^{\circ}$ whereas the obtained rough surface ascents to $155.8 \pm 2.84^{\circ}$, see representative images of water droplets over the surfaces on the top of panels A and B of Figure 1, respectively. The measured WCA did not change significantly with the deposition time of the drop, up to $1 \mathrm{~min}$.

Protein Adsorption Study. Low-protein adsorption materials are valuable in many areas (biosensors, biomaterials and devices that contact with blood, surfaces that cannot be often cleaned, labware, medical textiles, food packing, and so on) because they 


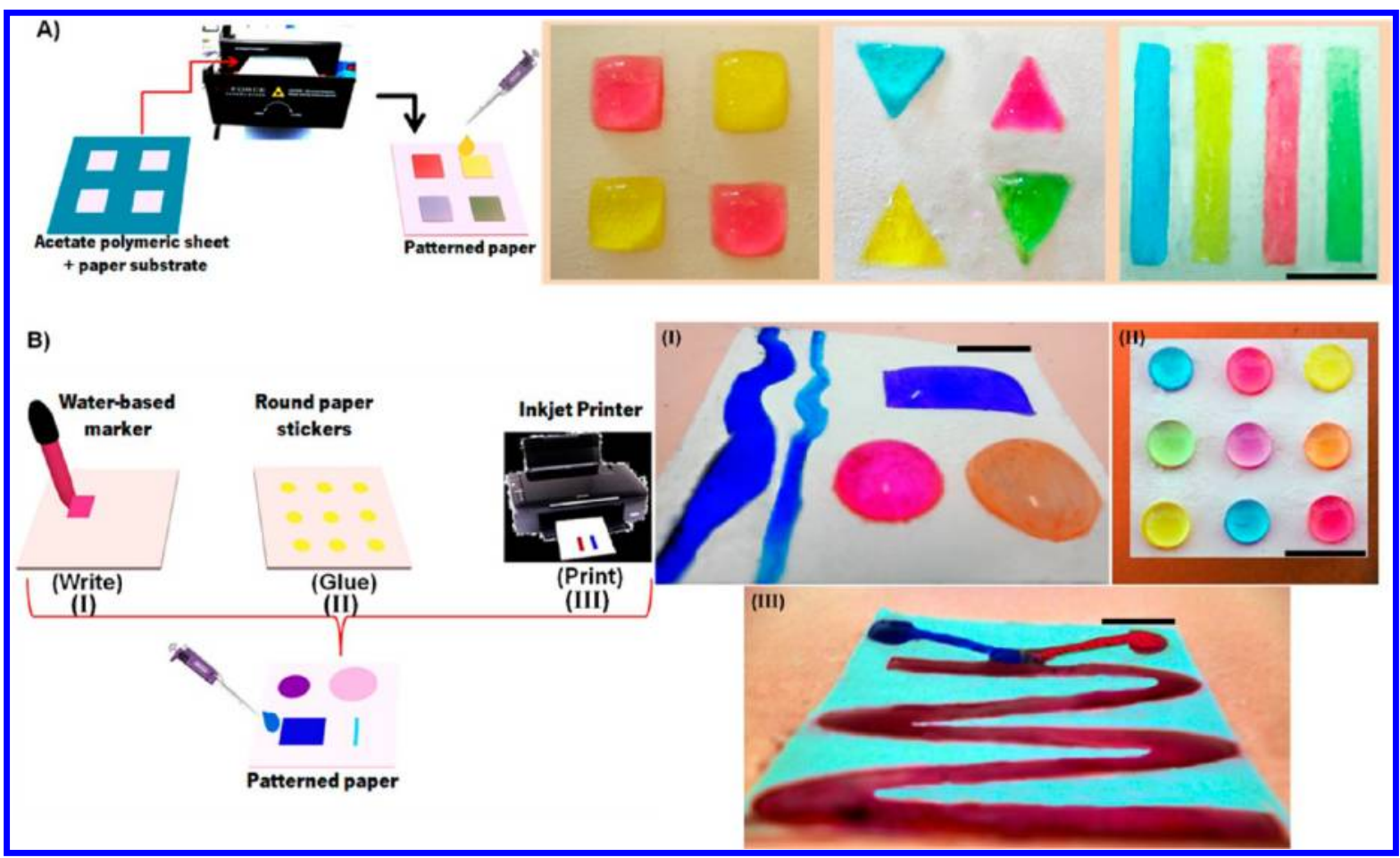

Figure 5. (A) Schematic representation of UVO patterning to create more wettable domains on flat SH substrates and the respective representative images with colored water; (B) schematic representation of handling and printing patterning to create more wettable domains on 2D superhydrophobic substrates and the respective representative images of patterning with (I) water-based marker, (II) round paper stickers and (III) inkjet printer, revealed by colored water. The scale bars are $10 \mathrm{~mm}$.

can reduce the biological contamination of surfaces. ${ }^{39,40}$ Previous works reported the influence of micro and nanotopographies on protein adsorption; ${ }^{41,42}$ however, just a small number of studies assessed protein adsorption on $\mathrm{SH}$ surfaces. ${ }^{39,43-45}$ We used BSA as a model biomolecule in the protein adsorption tests. As far as we know, this is the first protein adsorption study performed with water repellent paper surfaces.

Figure 2 shows the amount of adsorbed protein on both regular and $\mathrm{SH}$ paper after being exposed to BSA solutions of different concentrations. The results indicate that non wettable surfaces exhibit significant more resistance to protein adsorption than wettable paper surfaces, for the all range of initial concentrations explored. Such data are consistent with results previously obtained in other substrates ${ }^{43,44,46}$ and may be explained with the Cassie and Baxter model. ${ }^{47}$ In fact, if we consider that the air remains in the lower topographical regions of the surface, a decrease in the contact area between the surface and the protein solution will be expected.

Sterilization Effects. Lab-on-chip devices and labware often require to be sterilized, after which their properties should be preserved. ${ }^{48}$ To evaluate the post-sterilization behavior using ethylene oxide, the surface topography of $\mathrm{SH}$ paper was analyzed by SEM, see Figure 1C. No significant changes are detected when compared with the nonsterilized $\mathrm{SH}$ paper (Figure 1B). The WCA of post-sterilized SH paper samples was $151.8 \pm 1.81^{\circ}$, indicating that sterilization did not compromise the $\mathrm{SH}$ behavior of the substrate.

Wettability Modification. WCA data reveals the decrease in wettability of $\mathrm{SH}$ paper samples, with increasing exposure time to an UVO irradiation - see Figure 3. For example, for $30 \mathrm{~min}$ of UVO exposure the $\mathrm{SH}$ paper surface is switched to a very hydrophilic stage $\left(\mathrm{WCA}=11.4 \pm 3.5^{\circ}\right)$. Upon modification with UVO, we measured the WCA of the samples after different time points ( 1,5 , and 20 days), and during this period no significant recovery of hydrophobicity is observed.

The modified surfaces using UVO irradiation for 8 min was subjected to ethylene oxide sterilization. No significant differences could be detected in the WCA with the nonsterilized sample. Figure 4 shows the influence of sterilization on the WCA for the original paper, $\mathrm{SH}$ paper, and the $\mathrm{SH}$ paper irradiated with UVO, that show that ethylene oxide sterilization has a low influence on wettability. This is in a good agreement with the literature: ethylene oxide is described as a nonaggressive technique for a wide range of polymeric materials, with no significant change in their properties. ${ }^{49}$

3.2. Creation of Simple Two-Dimensional LOP Devices. As extensively reported by literature, the design of LOP devices are often based on the possibility to create different confined geometries onto the paper surface for fluidic flow or deposition of small liquid volumes. ${ }^{1,4,50}$ This is generally achieved by patterning the surface with more wettable domains. We demonstrate the possibility to pattern the $\mathrm{SH}$ paper surface using distinct approaches, each one with specific advantages, see Figure 5.

Using acetate sheets with hollowed geometrical features, it is possible to expose defined wettable domains of $\mathrm{SH}$ paper to UVO irradiation (Figure 5A). We choose the UVO treatment for $8 \mathrm{~min}$ to pattern the substrate because this irradiation time is sufficient to create a contrast of wettability but still prevents liquid penetration into the substrate. For the proof-of-concept we created standard geometries onto $\mathrm{SH}$ paper substrates, which are revealed when we dispensed colored water in the hydrophilic spaces, see schematic representation in Figure 5A. The UVO treated domains have well-defined limits and confine very efficiently the water volumes within the corresponding 


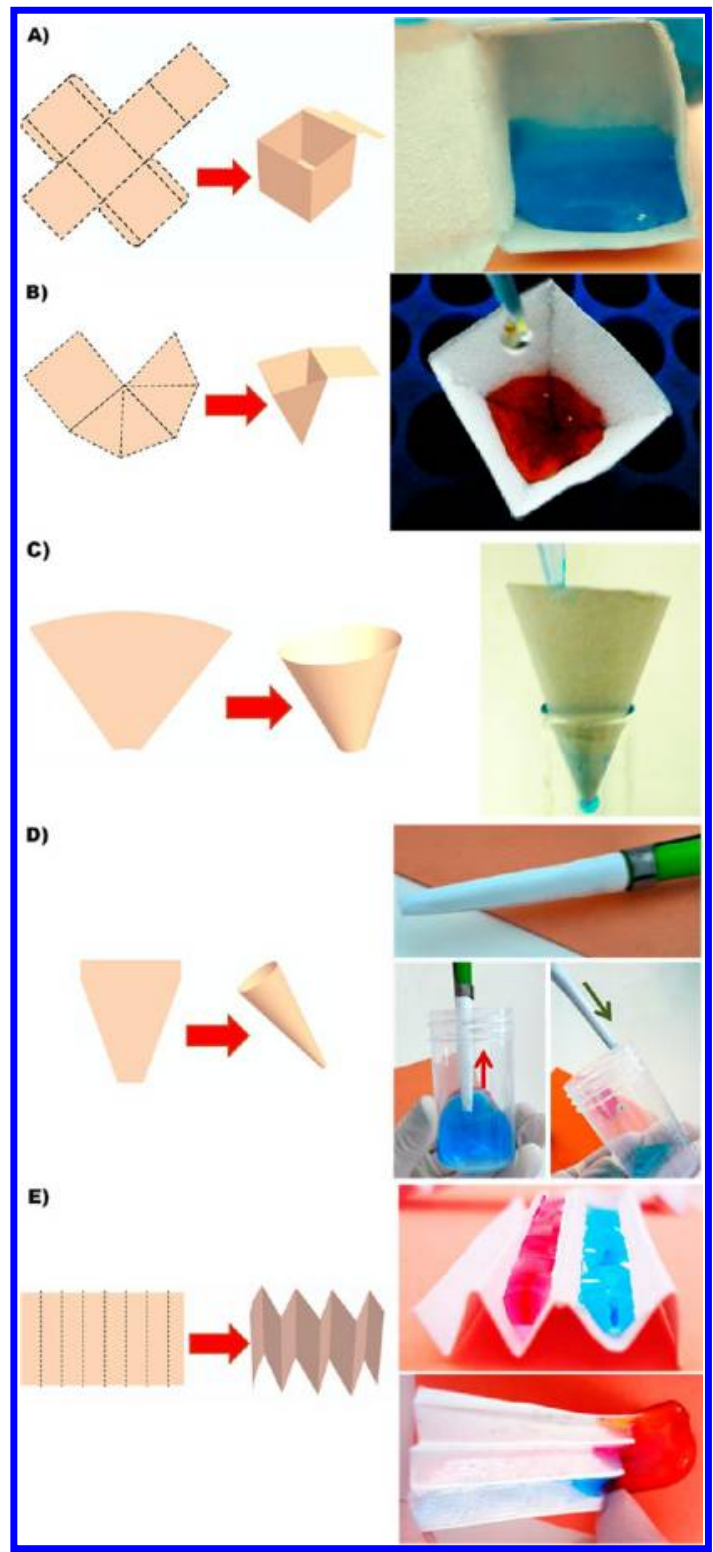

Figure 6. (A) Schematic representation of $3 \mathrm{D}$ SH paper cube to transfer, mix and store liquid samples and the respective representative image when dispensed colored water; (B) schematic representation of 3D SH paperlike eppendorf and the respective representative image when dispensed colored water; (C) schematic representation of $3 \mathrm{D}$ SH paper funnel and the respective representative image when dispensed colored water; (D) schematic representation of 3D $\mathrm{SH}$ paperlike tips and the respective representative images of the tip inserted in P1000 micropipet, the drawing up of the liquid solution and the transfer and dispensing on the target place; (E) schematic representation of 3D SH paper folded with an accordion shape for parallel liquid transfer and the respective representative images of the different channels covered by colored water solutions and the releasing of the liquids. The net of the 3D superhydrophobic structures has different areas, ranging between 1 and tens of square centimeters.

geometrical shapes, with minimum dimensions of about 500 $\mu \mathrm{m}$.

Easier to access and faster approaches were also investigated to pattern wettable regions over $\mathrm{SH}$ paper based on the fact that paper may be painted, glued or printed (see Figure 5B).

We first used a water-based marker to draw and paint the desired pattern geometry onto the surface of the $\mathrm{SH}$ paper

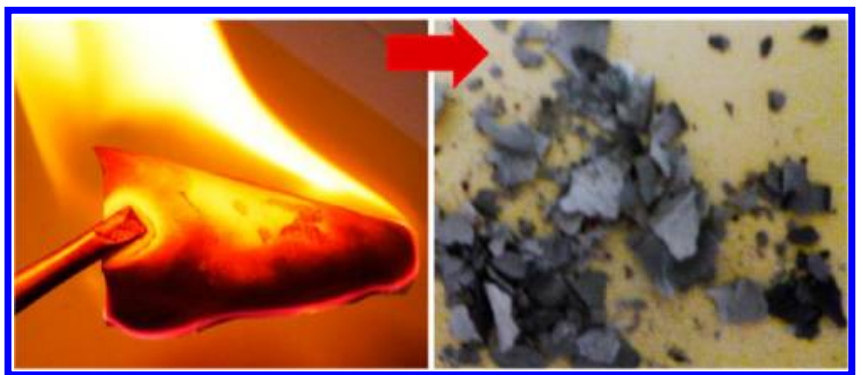

Figure 7. Representative image showing the possibility to incinerate the $\mathrm{SH}$ paper substrates up to an ash residue.

substrate, Figure 5BI. Also small paper stickers were glued to specific spots of the sample (Figure 5BII). With an easy-to-get marker or paper stickers is possible to coat single parts of the substrate with more wettable characteristics: WCA $=76.4 \pm$ $1.50^{\circ}$ for water-based marker and WCA $=82.9 \pm 4.28^{\circ}$ for paper stickers. Such elements provided the ability to confine liquid volumes with a controlled geometry, see schematic representation in Figure 5B. Both patterning processes involve straightforward benchtop methodologies and inexpensive resources. $^{20}$

For more complex patterning designs, we propose an inkjet printing process. We started to produce a pattern model in a computer and printed it directly on a $\mathrm{SH}$ paper piece, using standard colored inks. The WCA of the printed areas is $76.4 \pm$ $8.20^{\circ}$ and the resolution of the regions is defined by the specifications of the inkjet printer. After printing, the patterned $\mathrm{SH}$ paper is ready to be used and the confinement of liquids is consistent with the shape of the modified regions. Figure 5BIII shows an example of printed channels that could be used to produce substrates for open microfluidic applications. ${ }^{27}$ The good definition of the printed wettable domains (resolution around $100 \mu \mathrm{m}$ ), the simplicity and fastness of the procedure offer the possibility to produce at large scale low-cost LOP devices.

3.3. Construction of Three-Dimensional Superhydrophobic Paper Labware. The produced SH paper can be shaped, cut, folded, creased, wrapped, glued to form freestanding 3D architectures. As far as we aware, this is the first time that $\mathrm{SH}$ paper is suggested for 3D constructs. Low cost and disposable materials are interesting for labware industry. We propose different designs to alternative low-cost labware that take the advantage of the self-cleaning properties and water resistance of the developed material.

We describe two different structures, based on the folding of the $\mathrm{SH}$ paper substrates: a cube, which can be used in storing, transferring, and mixing biological or chemical samples (see Figure 6A); and an eppendorf-like object able to perform the same function as plastic ones, even when exposed to moderate centrifugal forces (Figure 6B). These materials can be produced in a wide range of sizes and can be easily written to identify the samples. The respective figures reveal the behavior of colored water in contact to the 3D assemblies: there is no adsorption of the sample at least for $24 \mathrm{~h}$.

Wrapping and glue $\mathrm{SH}$ paper also offers the possibility to fabricate useful structures in a simple way. Figure $6 \mathrm{C}$ shows a paper funnel with a $\mathrm{SH}$ inner surface, which can be used to transfer liquids. We dispensed colored water solution onto the object surface to verify its behavior. After continuous reuses, the inner face of the funnel was able to retain its ability to repeal water, suggesting that it can be used repeatedly to transfer 
liquids without the need of cleaning procedures. Micropipets are a daily used tool in laboratory procedures, using plastic tips to collect the liquid sample. We develop a SH paper tip, just by rolling up and glue the substrate, see Figure 6D. Paper porosity can be overcome by blocking the pores, using a polish. Therefore the vacuum action provides the required force to draw up precise measures of liquid, defined by the user, to be transferred and dispensed it to a target place. Again, the selfcleaning and the low protein adsorption of the $\mathrm{SH}$ paper permit to extend the reutilization of the tips, before being dispensed. Additionally, its low-cost and the possibility to produce at large scales suggest that $\mathrm{SH}$ paper tips could be an alternative to traditional plastic tips.

Inspired by the accordion shape, we use a zigzag folding of a single piece of $\mathrm{SH}$ paper to create one or more confined divisions or channels. When we dispense different liquid solution into these channellike structures the drops have tendency to roll off easily the surface to the end of the device, see Figure 6E. Such system may be useful to transport different liquids through distinct channel compartments, and can also be used to promote their mixture in the end, if a fanlike architecture is adopted.

The effect of water immersion with time was investigated, using 9 time points $(1,6,12,24$, and $48 \mathrm{~h}$, and $1,2,3$, and 4 weeks). During the first two weeks no significant changes were detected on the WCA value of the SH surface. After this time, the surface progressively loses its $\mathrm{SH}$ properties. The effect of the mechanical forces applied in the handling of the $\mathrm{SH}$ paper was also studied. No significant changes in the WCA were observed.

Especially when dealing with hazardous chemicals and biological liquids, substrates for diagnostics and on-site detection or for labware applications should be disposable after their use. Paper is a well-known biodegradable compostable material. Moreover, it can be readily eliminated by burning. Figure 7 shows that the developed SH paper may be easily incinerated up to an ash residue.

\section{CONCLUSIONS}

In conclusion, we have made use of different abilities of paper to create useful 2D and 3D structures based on $\mathrm{SH}$ paper. By tuning the wettability of confined geometries of the surface to hydrophilic, we developed simple and low cost procedures to pattern the SH surface with well-defined wettable regions; just by writing or printing we can produce a desirable patterning in a time scale of seconds or minutes. These results constitute an important step toward the development of completely flat LOP devices and substrates to manipulate liquid drops. SH paper substrates were also shown to be suitable to construct origamiderived 3D structures with promising applications by fixing the geometry using glue or by folding; we exploited the building of flexible, extremely low cost, and disposable alternative labware, providing the framework for a new way to use $\mathrm{SH}$ paper. Instead of acting like a final and target application, our results provide a springboard for the development of a wide range of new disposable LOP and labware devices.

\section{AUTHOR INFORMATION}

\section{Corresponding Author}

*E-mail: jmano@dep.uminho.pt.

\section{Notes}

The authors declare no competing financial interest.

\section{REFERENCES}

(1) Li, X.; Ballerini, D. R.; Shen, W. Biomicrofluidics 2012, 6, 113011130113.

(2) Bracher, P. J.; Gupta, M.; Whitesides, G. M. J. Mater. Chem. 2010, 20, 5117-5122.

(3) Hossain, S. M. Z.; Luckham, R. E.; Smith, A. M.; Lebert, J. M.; Davies, L. M.; Pelton, R. H.; Filipe, C. D. M.; Brennan, J. D. Anal. Chem. 2009, 81, 5474-5483.

(4) Zhao, W. A.; van den Berg, A. Lab Chip 2008, 8, 1988-1991.

(5) Macek, K.; Bečváŕová, H. Chromatogr. Rev. 1971, 15, 1-28.

(6) Li, M.; Tian, J.; Al-Tamimi, M.; Shen, W. Angew. Chem., Int. Ed. 2012, 51, 5497-5501.

(7) Li, X.; Tian, J.; Shen, W. Anal. Bioanal. Chem. 2010, 396, 495501.

(8) Martinez, A. W.; Phillips, S. T.; Whitesides, G. M.; Carrilho, E. Anal. Chem. 2009, 82, 3-10.

(9) Kim, J.; Yun, S.; Ounaies, Z. Macromolecules 2006, 39, 42024206.

(10) Fu, E.; Lutz, B.; Kauffman, P.; Yager, P. Lab Chip 2010, 10, 918-920.

(11) Balu, B.; Berry, A. D.; Hess, D. W.; Breedveld, V. Lab Chip 2009, 9, 3066-3075.

(12) Martinez, A. W.; Phillips, S. T.; Butte, M. J.; Whitesides, G. M. Angew. Chem., Int. Ed. 2007, 46, 1318-1320.

(13) Martinez, A. W.; Phillips, S. T.; Wiley, B. J.; Gupta, M.; Whitesides, G. M. Lab Chip 2008, 8, 2146-2150.

(14) Martinez, A. W.; Phillips, S. T.; Whitesides, G. M. Proc. Natl. Acad. Sci. U. S. A. 2008, 105, 19606-19611.

(15) Klasner, S.; Price, A.; Hoeman, K.; Wilson, R.; Bell, K.; Culbertson, C. Anal. Bioanal. Chem. 2010, 397, 1821-1829.

(16) Li, X.; Tian, J.; Shen, W. Cellulose 2010, 17, 649-659.

(17) Li, X.; Tian, J.; Nguyen, T.; Shen, W. Anal. Chem. 2008, 80, 9131-9134.

(18) Li, X.; Tian, J. F.; Garnier, G.; Shen, W. Colloids Surf., B 2010, $76,564-570$.

(19) Zhong, Z.; Wang, Z.; Huang, G. Microsyst. Technol. 2012, 18, 649-659.

(20) Lu, Y.; Shi, W.; Jiang, L.; Qin, J.; Lin, B. Electrophoresis 2009, 30, $1497-1500$

(21) Ogihara, H.; Xie, J.; Okagaki, J.; Saji, T. Langmuir 2012, 28, 4605-4608.

(22) Jin, C.; Jiang, Y.; Niu, T.; Huang, J. J. Mater. Chem. 2012, 22, $12562-12567$.

(23) Yang, H.; Deng, Y., J. Colloid Interface Sci. 2008, 325, 588-593.

(24) Balu, B.; Breedveld, V.; Hess, D. W. Langmuir 2008, 24, 47854790.

(25) Obeso, C. G.; Sousa, M. P.; Song, W.; Rodriguez-Pérez, M. A.; Bhushan, B.; Mano, J. F. Colloids Surf., A 2013, 416, 51-55.

(26) Freschauf, L. R.; McLane, J.; Sharma, H.; Khine, M. PLoS One 2012, 7, e40987.

(27) Oliveira, N. M.; Neto, A. I.; Song, W. L.; Mano, J. F. Appl. Phys. Express 2010, 3, 085205.

(28) Song, W.; Veiga, D. D.; Custódio, C. A.; Mano, J. F., Adv. Mater. (Weinheim, Ger.) 2009, 21, 1830-1834.

(29) Liu, H.; Crooks, R. M. J. Am. Chem. Soc. 2011, 133, 1756417566.

(30) Ge, L.; Yan, J.; Song, X.; Yan, M.; Ge, S.; Yu, J. Biomaterials 2012, 33, 1024-1031.

(31) Estridge, B. H.; Reynolds, A. P.; Walters, N. J. In Basic Medical Laboratory Techniques, 6th ed.; Delmar Publishers: Albany, NY, 2000; pp 5-95.

(32) Zhang, X.; Zhao, N.; Liang, S.; Lu, X.; Li, X.; Xie, Q.; Zhang, X.; Xu, J. Adv. Mater. 2008, 20, 2938-2946.

(33) Yuan, Z.; Chen, H.; Tang, J.; Chen, X.; Zhao, D.; Wang, Z. Surf. Coat. Technol. 2007, 201, 7138-7142.

(34) Wang, Y.; Liu, Z.; Han, B.; Sun, Z.; Zhang, J.; Sun, D. Adv. Funct. Mater. 2005, 15, 655-663.

(35) van de Witte, P.; Dijkstra, P. J.; van den Berg, J. W. A.; Feijen, J. J. Membr. Sci. 1996, 117, 1-31. 
(36) Zhao, N.; Xu, J.; Xie, Q.; Weng, L.; Guo, X.; Zhang, X.; Shi, L. Macromol. Rapid Commun. 2005, 26, 1075-1080.

(37) S.T, A.; P, B.; Richard, E.; Basu, B. J. Appl. Surf. Sci. 2012, 258, 3202-3207.

(38) Wei, Z. J.; Liu, W. L.; Tian, D.; Xiao, C. L.; Wang, X. Q. Appl. Surf. Sci. 2010, 256, 3972-3976.

(39) Koc, Y.; de Mello, A. J.; McHale, G.; Newton, M. I.; Roach, P.; Shirtcliffe, N. J. Lab Chip 2008, 8, 582-586.

(40) Zhang, H.; Lamb, R; Lewis, J. Sci. Technol. Adv. Mater. 2005, 6, 236-239.

(41) Scopelliti, P. E.; Borgonovo, A.; Indrieri, M.; Giorgetti, L.; Bongiorno, G.; Carbone, R.; Podestà, A.; Milani, P. PLoS One 2010, 5, e11862.

(42) Pegueroles, M.; Aparicio, C.; Bosio, M.; Engel, E.; Gil, F. J.; Planell, J. A.; Altankov, G. Acta Biomater. 2010, 6, 291-301.

(43) Lourenço, B.; Marchioli, G.; Song, W.; Reis, R.; van Blitterswijk, C.; Karperien, M.; van Apeldoorn, A.; Mano, J. F. Biointerphases 2012, 7, 1-11.

(44) Ballester-Beltran, J.; Rico, P.; Moratal, D.; Song, W.; Mano, J. F.; Salmeron-Sanchez, M. Soft Matter 2011, 7, 10803-10811.

(45) Neto, A. I.; Custodio, C. A.; Song, W.; Mano, J. F. Soft Matter 2011, 7, 4147-4151.

(46) Koch, K.; Barthlott, W. Philos. Trans. R. Soc. London, Ser. A 2009, 367, 1487-1509.

(47) Cassie, A. B. D.; Baxter, S. Trans. Faraday Soc. 1944, 40, 546551.

(48) Yeo, L. Y.; Chang, H.-C.; Chan, P. P. Y.; Friend, J. R. Small 2011, 7, 12-48.

(49) Massey, L. K. In The Effects of Sterilization Methods on Plastics and Elastomers the Definitive User'S Guide and Databook, 2nd ed.; William Andrew: Norwich, NY, 2005; pp 1-13.

(50) Ballerini, D.; Li, X.; Shen, W. Microfluid. Nanofluid. 2012, 13, 769-787. 